\title{
Análise da eficiência técnica de talhões de café irrigados e não-irrigados em Minas Gerais: 2004-2006
}

\author{
Vladimir Faria dos Santos ${ }^{2}$ \\ Wilson da Cruz Vieira ${ }^{3}$ \\ José Luís dos Santos Rufino ${ }^{4}$ \\ João Ricardo F. de Lima ${ }^{5}$
}

Resumo: Este trabalho teve como objetivo mensurar a eficiência técnica de 228 talhões de café das regiões do Cerrado e Sul de Minas Gerais e identificar seus principais determinantes, considerando-se lavouras irrigadas e não-irrigadas. Para mensurar a eficiência, utilizou-se a Análise Envoltória de Dados (DEA) e, para investigar seus determinantes, empregou-se um modelo Tobit. Os resultados obtidos mostram que a maioria das lavouras cafeeiras da amostra analisada apresenta ineficiência técnica, com destaque para as não-irrigadas.

Palavras-chaves: Produção de café, DEA, modelo Tobit, Minas Gerais.

Abstract: The objective of this study was to measure the technical efficiency of 228 plots of coffee from the regions of the Brazilian Savannas (Cerrado) and South of Minas Gerais and to identify the determining factors of that efficiency, considering irrigated and non-irrigated crops. The analyses were carried out using both Data Envelopment Analysis (DEA) and an econometric Tobit model. The results show that most of the coffee crops present technical inefficiency and among the most inefficient are the non-irrigated crops.

Key-words: Coffee production, DEA, Tobit model, Minas Gerais.

Classificação JEL: C24, C61.

${ }^{1}$ Os autores agradecem os comentários e as sugestões dos pareceristas.

${ }^{2}$ Economista. Doutorando em Economia Aplicada pela Universidade Federal de Viçosa. E-mail: vladi_fs@yahoo.com.br

${ }^{3}$ Professor Associado do Departamento de Economia Rural da Universidade Federal de Viçosa.E-mail: wvieira@ufv.br

${ }^{4}$ Pesquisador da Embrapa em Programa de Pós-Doutorado em Economia Aplicada na Universidade Federal de Viçosa. E-mail: jlsrufino@vicosa.ufv.br

${ }^{5}$ Professor Adjunto do Departamento de Ciências Fundamentais e Sociais da Universidade Federal da Paraíba. E-mail: jricardo@cca.ufpb.br 


\section{Introdução}

O café é uma das mais importantes commodities agrícolas e movimenta anualmente, no mercado internacional, mais de US\$ 60 bilhões (OLIVEIRA et al., 2005). O setor cafeeiro constitui importante atividade econômica, visto que gera renda e trabalho para milhares de pessoas nos países produtores. O Brasil, Vietnã e Colômbia, principais países produtores de café, produziram, em 2006, 69.112 mil sacas de $60 \mathrm{~kg}$, o que representa mais de $55 \%$ da produção mundial do grão (ABIC, 2007).

No Brasil, a economia cafeeira tem-se mostrado importante ao longo do tempo, mas sua participação relativa tem diminuído gradativamente, com a diversificação da economia brasileira. Apesar disso, o café continua sendo um produto de destaque, sobretudo pela geração de divisas. De acordo com o Conselho dos Exportadores de Café, Cecafé (2007), o Brasil embarcou, em 2006, cerca de 27,3 milhões de sacas de $60 \mathrm{~kg}$, gerando uma receita de quase US\$ 3,3 bilhões.

Minas Gerais é o maior produtor brasileiro do grão. Na safra 2006/2007, o estado produziu 21.987 mil sacas de $60 \mathrm{~kg}$, o que corresponde a $51 \%$ do total do País (CONAB, 2007). Dessa forma, o café tem contribuído, significativamente, para a geração de riqueza no estado mineiro. De acordo com o Banco de Desenvolvimento de Minas Gerais, o BDMG (2002), o setor cafeeiro participa com cerca de $26 \%$ do Produto Interno Bruto agropecuário de Minas Gerais.

Atualmente, a tecnologia da irrigação permite que o estado de Minas Gerais produza café de alta qualidade em regiões anteriormente consideradas impróprias devido ao elevado déficit hídrico, destacando-se o Triângulo Mineiro e o Alto Paranaíba. No Brasil, o uso da irrigação na cafeicultura é crescente estimativas indicam que essa técnica já atinge cerca de $10 \%$ da cafeicultura brasileira, totalizando 200.000 ha. Desse total, cerca de $25 \%$ situa-se em Minas Gerais (MANTOVANI, 2007; e EMBRAPA, 1999).

A irrigação apresenta muitas vantagens, entre estas, elevação da produção e da produtividade e decréscimo do risco climático (BERNARDO et al., 2005). Diante da elevada concorrência no setor cafeeiro e da constante oscilação dos preços, a eficiência produtiva pode ser considerada um dos fatores primordiais para que o cafeicultor permaneça em sua atividade. Assim, a análise da eficiência pode ser importante tanto para fins de planejamento, quanto para tomadas de decisões que visam uma melhor gestão.

Diante do exposto, objetivou-se, neste trabalho, investigar o desempenho de 228 talhões de café (irrigados e não-irrigados) das regiões do Cerrado e Sul de Minas Gerais e identificar aquelas lavouras que podem ser consideradas (in)eficientes em seus processos produtivos. Especificamente, buscou-se mensurar os graus de (in)eficiência técnica de unidades de produção irrigadas e não-irrigadas e identificar os principais determinantes dessa (in)eficiência. 


\section{Metodologia}

Para alcançar os objetivos propostos, utilizou-se, primeiramente, a técnica não-paramétrica Análise Envoltória de Dados (DEA) para mensurar a eficiência técnica e, em seguida, um modelo econométrico Tobit para identificar os fatores que determinam tal (in)eficiência.

Entende-se por eficiência a capacidade de alcançar objetivos por meio de uma relação desejável entre insumos e produtos ou, em outras palavras, a existência de máxima produtividade dos insumos empregados e/ou do mínimo custo na obtenção do produto (PASCUAL, 2000). A eficiência refere-se, portanto, ao modo como os agentes econômicos alcançam, da melhor forma possível, os objetivos de produção.

As medidas de eficiências são representadas, geralmente, por uma função de fronteira construída no sistema de coordenadas, sendo eficientes as unidades de produção que se posicionam sobre essa fronteira (FERREIRA, 2005). Nesse sentido, uma medida de eficiência é obtida ao mensurar a distância que uma firma se encontra da fronteira de produção.

\subsection{Mensuração da eficiência técnica}

A técnica não-paramétrica DEA, desenvolvida inicialmente por Charnes et al. (1978), tem o objetivo de mensurar a eficiência de unidades tomadoras de decisão, denominadas DMUs (Decision Making Units), na presença de inputs (recursos ou insumos) e outputs (produtos) comuns. De acordo com Charnes et al. (1978), esses outputs e inputs, normalmente, são múltiplos em caráter e podem também assumir diversas formas, por exemplo, medidas ordinais.

Essas eficiências, calculadas por meio da DEA, são obtidas pela programação matemática, na qual são construídas fronteiras de eficiências. É a partir dessas fronteiras que se verificam a eficiência relativa das DMUs e as medidas, se for o caso, a serem tomadas para se chegar a tal situação.

$\mathrm{Na}$ DEA há dois modelos clássicos: o $\mathrm{CCR}$ e o $\mathrm{BCC}^{6}$. O primeiro pressupõe tecnologias com retornos constantes à escala, enquanto o segundo pressupõe retornos variáveis à escala. Neste trabalho, foram utilizados os dois modelos, sendo aplicados com orientação insumo, isto é, dando ênfase na redução de insumos, sem, no entanto, comprometer o nível de produção.

\footnotetext{
${ }^{6}$ Os termos CCR e BCC derivam-se das iniciais de seus autores: Charnes, Cooper e Rhodes; e Banker, Charnes, Cooper, respectivamente.
} 
No que se refere ao modelo CCR, tem-se, segundo Coelli et al. (1998), a seguinte formulação matemática:

$$
\operatorname{MIN}_{\theta, \lambda} \theta
$$

$$
\begin{gathered}
\text { Sujeito a: }-y_{i}+Y \lambda \geq 0 \\
\theta x_{i}-X \lambda \geq 0 \\
\lambda \geq 0
\end{gathered}
$$

em que $\theta$ é um escalar, cujo valor será a medida de eficiência da i-ésima DMU. Caso o valor de $\theta$ seja igual a um, a DMU será considerada eficiente; caso contrário, será ineficiente. O parâmetro $\lambda$ é um vetor de constantes $n \times 1$, em que os valores são calculados para obter a solução ótima. Caso uma DMU seja eficiente, todos os valores de $\lambda$ serão zero; para uma DMU ineficiente, os valores de $\lambda$ serão os pesos utilizados na combinação linear de outras DMUs eficientes que influenciam a projeção da DMU ineficiente sobre a fronteira calculada; $y_{i}$ é um vetor $(m x 1)$ de quantidade de produtos da $i$-ésima DMU; $x_{i}$ é um vetor $(k x 1)$ de insumos da $i$-ésima DMU; Y é uma matriz de produtos, de dimensão $(n \times m)$; $X$ é uma matriz de insumos, de dimensão ( $n x k$ ). Nota-se que o problema de programação linear deve ser resolvido $n$ vezes, um para cada DMU, visto que $\theta$ deve ser obtido para cada DMU (COELLI et al., 1998).

O modelo com retornos constantes será apropriado apenas quando todas as firmas (DMUs) estiverem operando em escala ótima; todavia, em competição imperfeita, pode haver DMUs que não estejam operando nessas condições. Dessa forma, o uso do modelo CCR, quando nem todas as DMUs estiverem operando em escala ótima, resulta em medidas de eficiência técnica que se confundem com eficiência de escala. Assim, o uso do modelo BCC permite o cálculo das eficiências técnicas livres desses efeitos de escala (COELLI et al., 1998).

Para incorporar os retornos variáveis às DMUs analisadas, deve-se fazer uma reformulação no modelo CCR, incluindo uma restrição de convexidade. Essa restrição é a única diferença entre os modelos CCR e BCC. Portanto, de acordo com Coelli et al. (1998), o modelo com retornos variáveis à escala (BCC) pode ser representado da seguinte forma:

$\operatorname{MIN}_{\theta, \lambda} \theta$,

$$
\begin{array}{cl}
\text { Sujeito a: } & -y_{i}+Y \lambda \geq 0 \\
& \theta x_{i}-X \lambda \geq 0 \\
& N_{1}^{\prime} \lambda=1 \\
& \lambda \geq 0
\end{array}
$$

em que $N_{1}$ é um vetor $n \times 1$ de algarismos unitários. 
Por meio dos modelos CCR e BCC, é possível encontrar os valores de eficiência de escala para cada unidade analisada. Assim, segundo Coelli et al. (1998), se os valores de eficiência técnica encontrados nos dois modelos (CCR e BCC) forem diferentes, para dada DMU, ela terá ineficiência de escala. Ao contrário, se forem iguais, significa que a DMU estará operando na escala ótima. Algebricamente, pode-se calcular a eficiência/ineficiência de escala pela razão entre os escores de eficiência técnica com retornos constantes e variáveis:

$$
E S=\frac{E T_{C C R}}{E T_{B C C}},
$$

em que $E T_{C C R}$ é a eficiência técnica sob retornos constantes; $E T_{B C C}$ é a eficiência técnica sob retornos variáveis; e ES é a eficiência de escala.

De acordo com Coelli et al. (1998), uma falha dessa medida de eficiência de escala é que ela não indica se a firma (DMU) está operando na área de retornos crescentes ou retornos decrescentes. Para contornar isso, é necessário formular outro problema de programação linear, a qual é imposta a pressuposição de retornos não-crescentes; para isso, é necessário substituir, no modelo (2), a restrição $N_{1}^{\prime} \lambda=1$ pela restrição $N_{1}^{\prime} \lambda \leq 1$, isto é,

$$
\begin{gathered}
\operatorname{MIN}_{\theta, \lambda} \theta, \\
\text { Sujeito a: }-y_{i}+Y \lambda \geq 0, \\
\\
\theta x_{i}-X \lambda \geq 0, \\
N_{1}^{\prime} \lambda \leq 1 \\
\lambda \geq 0
\end{gathered}
$$

Nesse contexto, para identificar se a DMU está operando com retornos crescentes ou decrescentes, deve-se comparar o resultado encontrado para eficiência técnica, no modelo com retornos variáveis (RV), com o encontrado no modelo com retornos não-crescentes (RNC), isto é, se $\mathrm{ET}_{\mathrm{RNC}}=\mathrm{ET}_{\mathrm{RV}}$, implica retornos decrescentes. Alternativamente, se $\mathrm{ET}_{\mathrm{RNC}} \neq \mathrm{ET}_{\mathrm{RV}}$, implica retornos crescentes.

Mais importante do que verificar se uma DMU é eficiente, é encontrar os determinantes que a levaram a ocupar essa posição. Diante disso, utilizou-se, neste trabalho, um modelo econométrico para identificar quais fatores foram relevantes para explicar os níveis de eficiência. 


\subsection{Determinantes da (in)eficiência}

Os escores de eficiência no modelo DEA têm valores máximos iguais a 1 . Várias DMUs da amostra atingem esse valor; por isso, o regressor em um modelo para explicar a eficiência fica limitado a um teto igual a 1, ou seja, é truncado nesse valor (GONÇALVES et al., 2008). O modelo Tobit é uma alternativa aos Mínimos Quadrados Ordinários (MQO) e é empregado quando a variável dependente é truncada.

Nos anos recentes, o modelo Tobit tem sido utilizado conjuntamente com a análise DEA para verificar os determinantes da eficiência. Entre os trabalhos que utilizam esses dois modelos analíticos, citam-se os de Chilingerian (1995), Kirjavainen e Loikkanen (1998), Jackson e Fethi (2000), Fethi et al. (2002), Hoff (2007), entre outros.

De acordo com Greene (2003), a formulação geral do modelo Tobit é, normalmente, dada pela função índice:

$$
y_{i}^{*}=X_{i} \beta+\varepsilon_{i},
$$

em que $y_{i}^{*}$ é a variável-índice, também conhecida como variável latente; $X_{i}$ representa o vetor das variáveis explicativas; e $\beta$ é o vetor dos parâmetros a ser estimado. Assume-se que $\varepsilon_{i}$ seja normalmente distribuído, com média zero e variância constante, $\sigma^{2}$, ou seja, $\left(\varepsilon \sim N\left(0, \sigma^{2}\right)\right)$.

Apesar de o escore de eficiência possuir limite superior igual a um, Greene (1993) sugeriu usar a truncagem em zero. Portanto, o escore de eficiência da DEA é transformado e o valor truncado é concentrado em zero e isso pode ser feito usando a seguinte fórmula:

$$
y_{i}=(1 / \theta)-1
$$

O escore da DMU de eficiência igual a 1 é transformado para zero. Com essa transformação, os escores menores que 1 tornam-se valores positivos. Dessa forma, a equação estimada (5) revela quais as variáveis que reduzem a ineficiência.

Diante do exposto, o modelo Tobit padrão pode ser definido da seguinte forma, para a observação $i$ :

$$
\begin{aligned}
& \text { Se } y_{i}^{*}>0, \text { então } y_{i}=y_{i}^{*} \\
& \text { Se } y_{i}^{*} \leq 0, \text { então } y_{i}=0
\end{aligned}
$$


Normalmente, a técnica padrão para estimar o modelo Tobit é a da máxima verossimilhança. Assim, de acordo com Johnston e Dinardo (1997), para as observações em que $y^{*} \leq 0$, a contribuição da verossimilhança será dada por Prob $\left(y^{*} \leq 0\right)$, que é:

$$
\operatorname{Prob}\left(-X_{i} \beta \leq \varepsilon_{i}\right)
$$

Padronizando as variáveis em (7), obtém-se:

$$
\operatorname{Prob}\left(\frac{-X_{i} \beta}{\sigma} \leq \frac{\varepsilon_{i}}{\sigma}\right)=1-\Phi\left(\frac{X_{i} \beta}{\sigma}\right)
$$

Para uma observação $y^{*}>0$, a contribuição para a verossimilhança é:

$$
\operatorname{Prob}\left(y^{*}>0\right) \phi\left(y_{i}^{*} \mid y_{i}^{*}>0\right)=\Phi\left(\frac{X_{i} \beta}{\sigma}\right) \frac{1}{\sigma} \frac{\phi\left[\left(y_{i}-X_{i} \beta\right) / \sigma\right]}{\Phi\left(X_{i} \beta / \sigma\right)},
$$

em que $\Phi($ ) é a função de distribuição cumulativa normal padrão; e $\phi($ ) é a função de densidade probabilidade normal padrão.

Considerando esses dois componentes, pode-se formar a função de verossimilhança:

$$
L=\prod_{y_{i}=0}\left[1-\Phi\left(\frac{X_{i} \beta}{\sigma}\right)\right] \cdot \prod_{y_{i}=0} \frac{1}{\sqrt{2 \pi \sigma^{2}}} \exp \left[-\frac{1}{2} \frac{\left(y_{i}-X_{i} \beta\right)^{2}}{\sigma^{2}}\right]
$$

Logaritimizando a equação (10), tem-se a função de log-verossimilhança:

$$
L=\sum_{y_{i}=0} \ln \left[1-\Phi\left(\frac{X_{i} \beta}{\sigma}\right)\right]+\sum_{y_{i}>0}\left[\ln \frac{1}{\sqrt{2 \pi \sigma^{2}}}-\frac{1}{2} \frac{\left(y_{i}-X_{i} \beta\right)^{2}}{\sigma^{2}}\right],
$$

em que a primeira soma se refere ao conjunto de observações, no qual $y_{i}=0$, e a segunda, ao conjunto de observações, em que $y_{i}>0$.

No tocante ao efeito marginal, tem-se, segundo Greene (2003), a seguinte equação:

$$
E M_{x_{j}}=\frac{\partial E\left(y_{i}\right)}{\partial x_{j}}=\beta \Phi\left(\frac{x_{i} \beta}{\sigma}\right)
$$

O efeito marginal fornece, de forma geral, o impacto de cada variável na probabilidade de a DMU ser eficiente. 
Para avaliar o modelo Tobit como um todo, foi usado o teste de razão de verossimilhança (LR statistic). A interpretação desse teste é semelhante ao teste $F$ utilizado em modelos de mínimos quadrados ordinários, ou seja, pelo teste LR statistic verifica-se se as variáveis explicativas incluídas no modelo influência, conjuntamente, a variável dependente. O LR statistic é igual a 2 (FVLI - FVLR), em que FVLI é a função verossimilhança em $\log$ irrestrita, obtida por meio da regressão formada pelos coeficientes e pelo intercepto; e FVLR é a função verossimilhança em $\log$ com restrição, na qual se consideram todos os coeficientes de inclinação iguais a zero, mantendo apenas o intercepto. A estatística do teste segue a distribuição de qui-quadrado $\left(\chi^{2}\right)$, com graus de liberdade iguais ao número de coeficientes. As conclusões procedem da seguinte maneira: se LR $>\chi^{2}$ tabelado, implica que a regressão será válida como um todo, ou seja, haverá pelo menos uma variável explicativa inserida no modelo que exercerá algum tipo de influencia sobre a variável explicada.

\subsection{Fonte e tratamento dos dados}

Os dados foram obtidos do Educampo-Café, que é um projeto de desenvolvimento conduzido pelo Sebrae-MG, em parceria com treze associações de produtores de café (listadas no Quadro 1A do Anexo), que tem o objetivo de orientar grupos de produtores rurais, por meio da capacitação técnica e gerencial, sobre aspectos econômicos e sociais, tornando-os mais eficientes e competitivos.

Neste trabalho, foram utilizadas informações sobre a média de dois anos agrícolas consecutivos (2004/05 e 2005/06). Foram utilizados dados de 228 talhões de café das regiões do Cerrado e Sul de Minas Gerais, os quais foram divididos em duas subamostras: os que utilizam a irrigação na produção, que inclui 89 talhões com área média de 25,12 ha, e os que não utilizam essa tecnologia, que inclui 139 talhões com área média de 16,3 ha.

No enfoque do projeto Educampo-Café, o talhão refere-se a determinada área da lavoura cafeeira que pode ser considerada homogênea, por ter a mesma variedade de café plantada na mesma data, em um solo de características físicas, químicas e topográficas semelhantes, além de receber o mesmo manejo agronômico e o mesmo tratamento administrativo. $\mathrm{O}$ talhão é o Centro de Custo do Projeto, ou seja, é a unidade para a qual se registram dados, elaboram indicadores, efetuam as análises de desempenho e são propostas medidas de adequação ao âmbito do projeto Educampo-Café.

A metodologia empregada pelo projeto Educampo-Café prevê que um engenheiro agrônomo acompanhe entre 15 e 20 propriedades cafeeiras. $\mathrm{O}$ levantamento das informações é feito mediante a utilização de um programa de computador, elaborado para essa finalidade. 
Deve-se ressaltar que nas duas subamostras de talhões foi aplicado o modelo DEA, sendo os talhões considerados como DMUs neste trabalho.

Para aplicar o modelo DEA, foi considerado como output (Y) o Valor Bruto da Produção (VBP), enquanto os inputs ( $\left.\mathrm{X}^{\prime} \mathrm{s}\right)$ foram definidos com base no método I-O stepwise, de seleção de variáveis. Os resultados da aplicação deste método podem ser observados nos Quadros 2A a 9A, contidas no Anexo ${ }^{7}$. Na aplicação do método I-O stepwise consideraram-se, a princípio, cinco inputs, dos quais foram selecionados os seguintes:

- Mão-de-obra total envolvida na atividade (MDO), em R\$;

- Gasto com adubação (GA), em R\$;

- Gasto com colheita e pós-colheita (GC), em R\$; e

- Energia e combustível (EC), em R\$.

Em média, esses insumos correspondem, aproximadamente, a 78\% dos gastos realizados nos talhões da amostra.

As variáveis explicativas, incluídas no modelo econométrico, foram definidas de acordo com a sua importância no processo produtivo do café. Segundo Reis et al. (2005), a função de produção de uma lavoura de café é composta pelos seguintes insumos: terra, capital (máquinas, equipamentos e benfeitorias), mão-de-obra (fixa e temporária), custo de formação da lavoura e outros insumos (adubos, herbicidas, fungicidas e inseticidas). Com base nesse trabalho, definiram-se as seguintes variáveis:

- Produtividade da mão-de-obra, medida pela receita total/gastos com mão-de-obra;

- Produtividade da terra, medida pela produção/área plantada;

- Estoque de capital (máquinas, equipamentos e benfeitorias); e

- Produtividade do custo operacional efetivo, medida em produção total/custo operacional efetivo.

A variável produtividade do custo operacional efetivo foi inserida no modelo para retratar a sensibilidade dos custos referentes à formação da lavoura e aos custos com adubação, herbicidas, inseticidas, etc.

$\mathrm{O}$ custo operacional efetivo (COE) é composto pelos gastos referentes à adubação (que inclui adubos, herbicidas, fungicidas e inseticidas), à energia e combustível, à colheita e pós-colheita e à mão-de-obra, ou seja, esse custo retrata todos os desembolsos feitos com os talhões. ${ }^{8}$

\footnotetext{
${ }^{7}$ Para mais detalhes sobre o método I-O stepwise, ver Senra et al. (2007).

${ }^{8}$ No cálculo da variável produtividade do custo operacional efetivo foram subtraídos do COE os gastos com mão-de-obra, visto que essa variável já faz parte do modelo.
} 


\section{Resultados e discussão}

Com o intuito de facilitar o entendimento das análises que seguem, os resultados são expostos em duas seções. Na primeira, apresentam-se as eficiências técnica e de escala (modelos CCR e BCC) dos talhões de café e, na segunda, são enfatizados os determinantes da eficiência.

\subsection{Eficiência técnica}

Considerando-se a pressuposição de retornos constantes (modelo CCR) e variáveis (modelo BCC) e mediante a orientação insumo, foram obtidos os níveis (escores) de eficiência técnica e de escala de cada talhão da amostra. Cabe ressaltar que foram considerados eficientes os talhões que obtiveram escores de eficiência acima de 0,90 e ineficientes os que apresentaram níveis abaixo dessa medida. A justificativa para essa flexibilização, de acordo com Ferreira (2005), está no fato de se evitar prejuízo da análise, já que alguma DMU pode se destacar mais, em virtude de ser uma outlier e não de sua eficiência relativa.

Primeiramente, são apresentados os níveis de eficiência e as estatísticas descritivas referentes aos talhões em que não se utilizam a irrigação na produção de café (Tabela 1).

Tabela 1. Eficiência técnica dos talhões não-irrigados, sob condições de retornos constantes (RC) e retornos variáveis (RV), 2004/2006.

\begin{tabular}{cccc}
\hline $\begin{array}{c}\text { Escore de } \\
\text { eficiência }\end{array}$ & $\begin{array}{c}\text { Retornos } \\
\text { constantes }\end{array}$ & $\begin{array}{c}\text { Retornos } \\
\text { variáveis }\end{array}$ & $\begin{array}{c}\text { Eficiência } \\
\text { de escala }\end{array}$ \\
\hline Média & 0,6716 & 0,7620 & 0,8800 \\
Máximo & 1,0000 & 1,0000 & 1,0000 \\
Mínima & 0,1606 & 0,1724 & 0,4058 \\
Desvio padrão & 0,2027 & 0,2096 & 0,1278 \\
Talhões eficientes & 20 & 45 & 81 \\
$\%$ da amostra & 14,40 & 32,37 & 58,27 \\
\hline
\end{tabular}

Fonte: Resultados da Pesquisa.

Conforme a Tabela 1 , sob a pressuposição de retornos constantes, verifica-se que, do total de 139 talhões, apenas 20 são tecnicamente eficientes, o que corresponde a 14,4\% do total da amostra. A média de ineficiência técnica ficou em 0,3284 (1-0,6716), o que indica que, nos talhões tecnicamente ineficientes, há possibilidade de reduzir o uso dos insumos em $32,84 \%$, em média, mantendo-se, mesmo assim, o nível de produção. 
Considerando-se os retornos variáveis, nota-se que a medida de eficiência técnica média cresceu, aproximadamente, $13,5 \%$, passando de 0,6716 para 0,7620 . Essa diferença entre as médias indica que 9 pontos percentuais, dos 32,37\% de ineficiência técnica (modelo CCR), são devidos à ineficiência de escala. Ademais, ao considerar o modelo BCC, o número de talhões eficientes passou para 45, um crescimento de $125 \%$. Isto ocorreu porque toda DMU (talhão) considerada tecnicamente eficiente, sob retornos constantes, também foi eficiente sob retornos variáveis (SEIFORD e ZHU, 1999), entretanto, o inverso não ocorreu. Além disso, o modelo BCC não leva em consideração a existência de ineficiência de escala.

A medida de eficiência de escala reflete a relação entre as medidas de eficiência técnica de retornos constantes e as com retornos variáveis. A DMU será considerada eficiente quando ela operar em escala ótima de produção. Diante disso, constata-se, de acordo com a Tabela 1 , que mais da metade $(58,27 \%)$ dos talhões de café está na escala ótima de produção. Cabe enfatizar que o conceito de escala ótima, utilizada neste trabalho, não se refere, necessariamente, ao ponto de mínimo custo médio de longo prazo, mas, sim, a retornos constantes à escala.

Embora tenha sido constatado que $41,73 \%$ dos talhões apresentam ineficiência de escala, estes podem exibir retornos crescentes ou decrescentes, podendo-se identificá-los formulando um problema de programação linear, na qual é imposta a pressuposição de retornos não-crescentes à escala no modelo BCC. Os resultados podem ser observados na Tabela 2.

Tabela 2. Distribuição dos talhões não-irrigados segundo o tipo de retornos, 2004/2006.

\begin{tabular}{ccc}
\hline Escala de produção & Número de talhões & $\%$ \\
\hline Retornos crescentes & 26 & 18,7 \\
Retornos constantes & 81 & 58,2 \\
Retornos decrescentes & 32 & 23,1 \\
Total & 139 & 100 \\
\hline
\end{tabular}

Fonte: Resultados da pesquisa.

Entre os talhões que apresentam ineficiências de escala, 26 estão operando na região de retornos crescentes, o que significa dizer que $18,7 \%$ destes estariam em melhor situação, em termos de eficiência, caso a escala de produção fosse maior. Entretanto, é oportuno destacar que o tamanho da área de um talhão é fixo ${ }^{9}$, o

\footnotetext{
${ }^{9}$ Como o café é um cultivo permanente e, por definição, o talhão é uma área geográfica determinada que foi plantada ao mesmo tempo, o tamanho físico de um talhão só se altera com a substituição de toda a lavoura, o que é inviável de se fazer no curto prazo. Por isso, considera-se o tamanho de um talhão como fixo.
} 
que impossibilita o aumento da escala de produção via crescimento da área plantada. Assim, só é possível mudar a escala de produção de um talhão se for alterada sua produtividade.

$\mathrm{Na}$ região de retornos decrescentes estão 32 talhões, o que corresponde a $23,1 \%$ do total. Esse resultado mostra que o nível de eficiência seria maior, se esses talhões possuíssem escala menor de produção.

Finalmente, 81 talhões estão operando na região de retornos constantes, isto é, são escala eficientes. Nota-se que esse número é igual ao número de talhões que alcançaram a eficiência de escala (Tabela 1), o que é explicado pelo fato de a eficiência de escala retratar a escala ótima de produção, que, por sua vez, ocorre na faixa de retornos constantes.

Ao considerar os talhões que utilizam no processo de produção a tecnologia de irrigação, têm-se os resultados apresentados na Tabela 3.

Tabela 3. Eficiência técnica dos talhões irrigados, sob condições de retornos constantes (RC) e retornos variáveis (RV), 2004/2006.

\begin{tabular}{cccc}
\hline $\begin{array}{c}\text { Escore de } \\
\text { eficiência }\end{array}$ & $\begin{array}{c}\text { Retornos } \\
\text { constantes }\end{array}$ & $\begin{array}{c}\text { Retornos } \\
\text { variáveis }\end{array}$ & $\begin{array}{c}\text { Eficiência } \\
\text { de escala }\end{array}$ \\
\hline Média & 0,6777 & 0,7247 & 0,9261 \\
Máximo & 1,0000 & 1,0000 & 1,0000 \\
Mínima & 0,1899 & 0,2290 & 0,5153 \\
Desvio padrão & 0,1972 & 0,1988 & 0,1056 \\
Talhões eficientes & 15 & 21 & 66 \\
\% da amostra & 16,8 & 23,6 & 74,2 \\
\hline
\end{tabular}

Fonte: Resultados da pesquisa.

O nível médio de ineficiência com retornos constantes foi de 0,3223 , o que significa que, em média, os talhões ineficientes podem reduzir seus gastos com insumos em $32,23 \%$, mantendo o mesmo nível de produção. Já a medida de eficiência técnica média, considerando-se retornos variáveis à escala, sobiu para 0,7247. Como enfatizado anteriormente, esse valor foi maior do que o referente ao modelo CCR devido ao fato de o modelo BCC não levar em consideração a existência de ineficiência de escala. No quesito eficiência de escala, $74,2 \%$ dos talhões (66) que utilizam a irrigação na plantação de café estão operando em escala ótima de produção.

Com relação à escala, têm-se os seguintes resultados referentes aos talhões irrigados (Tabela 4). 
Tabela 4. Distribuição dos talhões irrigados, segundo os tipos de retornos, 2004/2006.

\begin{tabular}{ccc}
\hline Escala de produção & Número de talhões & $\%$ \\
\hline Retornos crescentes & 16 & 18,0 \\
Retornos constantes & 66 & 74,2 \\
Retornos decrescentes & 7 & 7,8 \\
Total & 89 & 100 \\
\hline
\end{tabular}

Fonte: Resultados da pesquisa.

Conforme Tabela 4, observa-se que, do total de 89 talhões, 16 estão na parte da fronteira de produção que apresenta retornos crescentes, o que indica que $18 \%$ deles seriam mais eficientes, caso a escala de produção fosse maior.

No que se refere à região decrescente, verifica-se que há apenas 7 talhões nessa faixa, o que corresponde a 7,8\% do total. Este grupo, portanto, está operando acima da escala ótima de produção. Por fim, 66 talhões $(74,2 \%)$ estão na faixa de retornos constantes, isto é, encontram-se na escala ótima de produção.

Diante desses resultados, observa-se que o melhor desempenho foi dos talhões irrigados, ou seja, ao comparar os resultados da Tabelas $1 \mathrm{com}$ a 3 , percebe-se que o número de talhões eficientes, no quesito eficiência técnica (retornos constantes) e eficiência de escala, foi maior (proporcionalmente) nos irrigados. Esse resultado superior, alcançado pelos talhões irrigados, pode ser explicado, principalmente, pelos benefícios que o sistema de irrigação proporciona, como, por exemplo, otimizar o uso dos fertilizantes, proporcionar a redução do risco na agricultura e possibilitar trabalhos em escala na colheita (BERNARDO et al., 2005). Além disso, o tamanho físico das lavouras irrigadas, normalmente, é maior, o que proporciona ganhos de redução do custo médio por hectare irrigado; em outras palavras, com uma área maior, é possível utilizar os insumos com maior eficiência, sobretudo os relacionados com máquinas e equipamentos e com a aplicação de fertilizantes e defensivos.

\subsection{Determinantes da (in)eficiência}

Após obter os escores de eficiência, por meio da DEA, utilizou-se um modelo econométrico de amostra censurada (Tobit) para investigar as variáveis que determinam a eficiência (ineficiência) dos talhões. Foram utilizados, como variável dependente, os escores obtidos por meio do modelo CCR. Essa escolha se deu em razão de o modelo CCR ter maior precisão na discriminação da eficiência, visto que, para que uma DMU seja considerada tecnicamente eficiente no modelo com retornos constantes à escala, é necessário que ela seja tecnicamente eficiente no modelo com retornos variáveis; entretanto, o contrário não ocorre.

Antes de analisar os resultados, é importante ressaltar a possibilidade de que as variáveis relacionadas com produtividade estejam correlacionadas com o termo de 
erro do modelo. Caso as variáveis fossem endógenas, o modelo Tobit padrão não poderia ser aplicado. A suspeita de endogeneidade foi avaliada por meio do teste de exogeneidade das variáveis instrumentalizadas, de Wald, e por meio do teste de endogeneidade, de Hausman. ${ }^{10}$ No primeiro, se a estatística fosse significativa, havia possibilidade de considerar as variáveis instrumentalizadas como endógenas; no segundo, testa-se a hipótese nula de que há diferença estatística nos coeficientes estimados pelo modelo Tobit, com variáveis instrumentalizadas, e pelo modelo Tobit padrão. Se a hipótese nula não fosse rejeitada, as variáveis (regressores) poderiam ser consideradas como exógenas e o modelo padrão poderia ser utilizado. Para realizar o teste foi necessário definir três variáveis instrumentais (uma para cada possível variável endógena), a saber: mão-de-obra, custo operacional efetivo por área e área plantada.

Os resultados do teste de Wald e de Hausman, que podem ser observados nas Tabelas 1A e 2A do Anexo, revelam que os regressores são exógenos; portanto, é possível aplicar o modelo Tobit padrão.

Os resultados referentes ao modelo Tobit, considerando os talhões não-irrigados, podem ser observados na Tabela 5.

Tabela 5. Fatores associados à eficiência técnica dos talhões não-irrigados, 2004/2006.

\begin{tabular}{cccc}
\hline Variáveis & Efeito marginal & Erro-padrão & P-valor \\
\hline Prod. MDO & $-0,06496$ & 0,01788 & 0.000 \\
Prod. Terra & -0.01393 & 0,00789 & 0.077 \\
Capital & $-2,21 \mathrm{e}-07$ & $1,30 \mathrm{e}-06$ & 0.864 \\
Prod. COE & 1,277937 & 8,34460 & 0.878 \\
\hline
\end{tabular}

Ln da Função de Verossimilhança irrestrita $=-181,21$

Ln da Função de Verossimilhança Restrita

(somente com intercepto) $=-196,04$

$\chi^{2}$ calculado $($ LR) $=29,66$

Fonte: Resultados da pesquisa.

Prod. MDO = produtividade da mão-de-obra; Prod. Terra = produtividade da terra; Prod. COE = Produtividade do custo operacional efetivo.

Por meio do teste de Razão de Verossimilhança, verifica-se que esse modelo pode ser considerado globalmente válido, já que seu valor $(29,66)$ foi superior ao $\chi^{2}$ tabelado $(13,08)$, a $1 \%$ de significância, o que indica que os coeficientes estimados apresentaram, conjuntamente, ajustamento satisfatório.

Apesar de os coeficientes estimados exercerem, conjuntamente, influência na variável dependente (escore de eficiência técnica), dois deles não foram significativos: capital e produtividade do custo operacional efetivo (Prod. COE). O primeiro pode ser justificado pelo fato de as propriedades que não utilizam

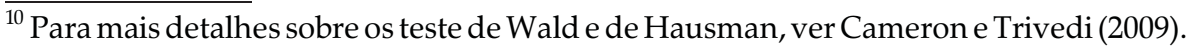


irrigação na produção de café não serem intensivas em capital, comparadas com as propriedades que utilizam tal tecnologia. Dessa forma, constata-se que o capital não é um fator importante para explicar a eficiência (ineficiência) técnica dos talhões não-irrigados.

Da mesma forma que o capital, a variável Prod. COE também não foi significativa, o que sugere que a produtividade do custo operacional efetivo não é um fator preponderante para explicar a ineficiência dos talhões que não utilizam a irrigação em sua plantação.

Ao contrário das variáveis Prod. COE e Capital, os coeficientes relacionados com produtividade da mão-de-obra e produtividade da terra foram significativos a $1 \%$ e $10 \%$, respectivamente. Ambas as variáveis contribuem, positivamente, para a eficiência técnica dos talhões não-irrigados.

O efeito marginal, associado à produtividade da mão-de-obra, indica que o aumento nos gastos com mão-de-obra em R \$1,00 (um Real) está ligado à queda da ineficiência técnica em 0,065 , o que reflete a importância da mão-de-obra para a eficiência dos talhões não-irrigados. No que se refere ao efeito marginal da produtividade da terra, tem-se o valor de -0.014 , que indica que, se houvesse aumento na produtividade da terra, haveria queda na ineficiência.

Os resultados associados aos talhões que utilizam irrigação na produção de café podem ser observados na Tabela 6.

Tabela 6. Fatores associados à eficiência técnica dos talhões irrigados, 2004/2006.

\begin{tabular}{cccc}
\hline Variáveis & Efeito marginal & Erro-padrão & P-valor \\
\hline Prod. MDO & $-0,0554817$ & 0,0195504 & 0.005 \\
Prod. Terra & 0,0024141 & 43,45329 & 0.660 \\
Capital & $-1,71 \mathrm{e}-06$ & $5,75 \mathrm{e}-07$ & 0.003 \\
Prod. COE & $-188,3126$ & 0,0054823 & 0.000 \\
\hline
\end{tabular}

Ln da Função de Verossimilhança irrestrita $=-71,21$

Ln da Função de Verossimilhança Restrita

(somente com intercepto) $=-102,56$

$\chi^{2}$ calculado $(\mathrm{LR})=62,7$

Fonte: Resultados da pesquisa.

No caso da questão da exogeneidade, aplicou-se o teste de Wald e de Hausman; no teste de Wald, rejeitou-se a hipótese de exogeneidade; porém, ao considerar o teste de Hausman, a hipótese de que as variáveis são exógenas não foi rejeitada. Dado o resultado do teste de Hausman, optou-se por considerar os regressores como exógenos. Os testes podem ser observados nas Tabelas 3A e 4A, do Anexo. 
Nota-se que o modelo como um todo apresenta significância estatística, visto que o $\chi^{2}$ calculado, do teste de Razão de Verossimilhança, foi significativo a $1 \%\left(\chi^{2}\right.$ tabelado $\left.=13,08\right)$.

Quanto à significância dos coeficientes, todos eles foram significativos a 1\%, exceto a variável produtividade da terra, ou seja, a produtividade da mão-de-obra, a do custo operacional efetivo e o estoque de capital são variáveis que reduzem a ineficiência técnica dos talhões irrigados. Cabe enfatizar o forte impacto que a variável Prod. COE exerce na (in)eficiência dos talhões, já que seu coeficiente foi de -188,3. Quanto maior a produção por custo operacional efetivo, menor tende a ser o nível de ineficiência.

Um ponto importante, que merece destaque, é o valor não-significativo do coeficiente da variável Prod. Terra, diferentemente da análise com os talhões não-irrigados. Esse resultado está ligado ao fato de os talhões dessa segunda subamostra utilizar a irrigação na sua lavoura. Por usarem essa tecnologia, as produtividades dos talhões tendem a ser maiores e a um nível semelhante entre eles, o que faz supor que a produtividade da terra não é um fator que exerce influência no nível de eficiência/ineficiência técnica.

\section{Considerações Finais}

Este trabalho analisou a eficiência técnica de 228 talhões de café localizados nas regiões do Cerrado e Sul de Minas Gerais, bem como os fatores que influenciam a variação dos escores de (in)eficiência. Os talhões foram divididos em duas subamostras: os que utilizam irrigação na produção (89) e os que não utilizam a irrigação (139).

Os principais resultados evidenciaram que há predomínio dos talhões (tanto os irrigados como os não-irrigados) com ineficiência técnica (escores de eficiência inferior a 0,9), ou seja, na maioria dos talhões há margem para redução dos insumos sem, no entanto, comprometer o nível de produção. Embora se esperasse um número significativo de talhões eficientes, devido à orientação técnica e gerencial recebida pelos produtores da amostra, o que prevaleceu foi o contrário. As razões disso podem estar associadas, por exemplo, às limitações financeiras e/ou educacionais dos produtores. Esses fatores impediriam a implementação e/ou assimilação efetiva das orientações fornecidas pelo projeto Educampo.

Diante da constatação de tal fato (nível elevado de ineficiência), torna-se necessário, então, contornar esse problema. Um das maneiras para solucionar esse cenário seria atuar sobre as variáveis que impactam o nível de (in)eficiência técnica. No caso dos talhões não-irrigados, merecem atenção as variáveis produtividade da mão-de-obra e da terra; já os irrigados merecem destaque a produtividade da mão-de-obra, o estoque de capital e a produtividade do custo operacional efetivo. 
Ademais, é interessante ressaltar a importância da irrigação. Nos talhões irrigados, a produtividade da terra tende a ser maior do que nos talhões que não utilizam essa técnica. A irrigação, portanto, gera alta produção por unidade de área plantada, ou seja, mesmo os talhões que não utilizam os insumos de forma eficiente tendem a ter alta produtividade, devido à tecnologia da irrigação. Dessa forma, a produtividade da terra (em áreas irrigadas) não seria um foco de ineficiência técnica e, assim, justificaria a não significância da variável Prod. Terra na segunda subamostra. Ao contrário, na primeira subamostra, na qual os talhões não utilizam a irrigação e a produtividade da terra (comparada aos talhões irrigados) é menor, a variável Prod. Terra mostrou-se importante para reduzir a ineficiência. $^{11}$

\section{Referências Bibliográficas}

ASSOCIAÇÃO BRASILEIRA DA INDÚSTRIA DE CAFÉ (ABIC). Estatística. Disponível em: < http://www.abic.com.br/estat_exportacoes.html >. Acesso em: 14 de junho de 2007.

BANCO DE DESENVOLVIMENTO DE MINAS GERAIS - BDMG. minas Gerais do Século XXI - Volume I. Belo Horizonte, 2002. 243p.

BERNARDO, S.; SOARES, A. A.; MANTOVANI, E. A. Manual de irrigação. $7^{a}$ ed. Viçosa: UFV, 2005. 625 p.

CAMERON, A. C.; TRIVEDI, P. K. Microeconometrics using stata. College Station, Texas: Stata Press Publication, 2009. 692 p.

COMPANHIA NACIONAL DE ABASTECIMENTO - CONAB. Central de Informações Agropecuárias. Disponível em: http://www.conab.gov.br/conabweb/ download/safra/2_levantamento_200708.pdf Acesso em: 01 Out. 2007.

CONSELHO DOS EXPORTADORES DE CAFÉ - CECAFÉ. Dados estatísticos. Disponível em: < http://www.cecafe.com.br> . Acesso em: 02 de Agosto de 2007.

CHARNES, A.; COOPER, W.W.; RHODES, E. Measuring the efficiency of decision-making units. European Journal of Operational Research, v. 2, p. 429-444, 1978.

CHILINGERIAN, J. A. Evaluating physician efficiency in hospitals: A multivariate analysis of best practices. European Journal of Operational Research, v. 80, p.548-574, 1995.

\footnotetext{
${ }^{11}$ A média da produtividade da terra dos talhões irrigados e não-irrigados é, de acordo com a amostra, de 35 sacas/ha e de 31 sacas/ha, respectivamente.
} 
COELLI, T.; RAO, D.S.P.;BATTESE, G. E. An introduction to efficiency and productivity analysis. London: Kluwer Academic, 1998. 275 p.

EMPRESA BRASILEIRA DE PESQUISA AGROPECUÁRIA - EMBRAPA. Relatório da estimativa da safra cafeeira no Brasil safra 1999/2000. Consócio Brasileiro de Pesquisa e Desenvolvimento do Café - Embrapa. 6 p. 1999

FERREIRA, M. A. M. Eficiência técnica e de escala de cooperativas e sociedades de capital na indústria de laticínios do Brasil. $151 \mathrm{f}$. Tese (Doutorado em Economia Aplicada) - Universidade Federal de Viçosa, Viçosa, MG, 2005.

FETHI, M.D.; JACKSON, P.M.; WEYMAN-JONES, T.G. Measuring the efficiency of European airlines: An application of DEA and tobit analysis. Discussion Paper, University of Leicester, 2002.

GONÇALVES, R. M. L.; VIEIRA, W. C.; LIMA, J. E.; GOMES, S. T. Analysis of technical efficiency of Milk-producing farms in Minas Gerais. Revista de Economia Aplicada. v. 12, n. 2 p. 321-335, 2008.

GREENE, W. H. Econometric analysis. 5. ed. New Jersey: Prentice Hall, 2003. $1.026 \mathrm{p}$.

GREENE, W.H. The econometric approach to efficiency analysis, in H.Fried, C.A.K. Lovel, and S.S. Schmidt (eds.), The measurement of productive efficiency and application, New York, Oxford University Press, 1993.

HOFF, A. Second stage DEA: Comparison of approaches for modelling the DEA score. European Journal of Operational Research, v. 181, p. 425-435, 2007.

JACKSON, P. M; FETHI, M. D. Evaluating the technical efficiency of Turkish commercial banks: An Application of DEA and Tobit Analysis. In. INTERNATIONAL DEA SYMPOSIUM, University of Queensland, Brisbane, Austrália, 2000.

JOHNSTON, J.; DINARDO, J. Econometric Methods. New York: McGraw Hill, 1997 531p.

KIRJAVAINEN, T.; LOIKKANEN, H. A. Efficiency Differences of Finnish Sênior Secondary Schools: An Application of DEA and Tobit Analysis. Economics of Education Review, 17, 4, 377-394, 1998.

MANTOVANI, E. C. A irrigação do cafeeiro. Disponível em < http://www.sbicafe.ufv.br/PDF/Conteudo/155552_Art01f.PDF>. Acesso em: 18 de junho de 2007.

OLIVEIRA, M.D.M.; VEIGA FILHO, A. A.; VEGRO, C.L.R.; MATTOSINHO, P.S.V.; MORICOCHI, L. Investimento e rentabilidade na produção de café especial: um estudo de caso. Informações Econômicas. V.35, No. 9, 2005. 
PASCUAL, R. F. Eficiencia de los centros públicos de educación secundaria de la provincia de Alicante. 237 f. Tese (Doctorado en Ciências econômicas) Universidad de Alicate, Alicate, 2000.

REIS, R. P.; RICHETTI, A.; LIMA, A. L. R. Eiciência econômica na cultura do café: um estudo no sul de Minas Gerais. Organizações rurais e agroindustriais. v. 7, n.1, p. 50-59, 2005.

SEIFORD, L. M.; ZHU, J. An investigation of returns to scale in data envelopment analysis. Omega: The journal of management science. v.27, n.1, p. 1-11, 1999. 
696 - Análise da eficiência técnica de talhões de café irrigados e não-irrigados em Minas Gerais: 2004-2006

\section{Anexo}

Quadro 1A. Associações de produtores de café que fazem parte do projeto Educampo-Café.

\begin{tabular}{|l|l|}
\hline ACA & Associação dos Cafeicultores de Araguari \\
\hline Amoca & Associação dos Cafeicultores da Região de Monte Carmelo \\
\hline Capal & Cooperativa Agropecuária de Araxá Ltda \\
\hline Acanor & Associação dos Cafeicultores do Noroeste Mineiro \\
\hline Assocafé & Associação dos Cafeicultores de Carmo do Paranaíba \\
\hline Assogotardo & Associação dos Cafeicultores de São Gotardo \\
\hline Cocatrel & Cooperativa dos Cafeicultores da Zona de Três Pontas \\
\hline Coopa & Cooperativa Agropecuária de Patrocínio \\
\hline Cooparaiso & Cooperativa Regional dos Cafeicultores de São Sebastião do Paraíso \\
\hline Cooxupé & Cooperativa Regional dos Cafeicultores de Guaxupé \\
\hline Copermonte & Cooperativa Agrícola de Monte Carmelho Ltda \\
\hline Expocaccer & Associação dos Cafeicultores do Cerrado \\
\hline Unicoop & Cooperativa Agropecuária do Sul de Minas \\
\hline
\end{tabular}

Fonte: Educampo-Café.

Quadro 2A. Escolha da primeira variável, talhões não-irrigados - I-O Stepwise.

\begin{tabular}{|c|c|c|c|c|c|}
\hline Input & GC & EC & GA & MDO & GM \\
\hline Output & VBP & VBP & VBP & VBP & VBP \\
\hline Média da eficiência & 0,2540 & 0,2046 & 0,3123 & $\mathbf{0 , 4 5 5 0}$ & 0,0288 \\
\hline
\end{tabular}

Fonte: Resultados da Pesquisa.

Nota: GM = Gasto com Manejo (controle de pragas e doenças; e controle de plantas daninhas), em R\$

Quadro 3A. Escolha da Segunda variável, talhões não-irrigados - I-O Stepwise.

\begin{tabular}{|c|c|c|c|c|}
\hline Input & GC & EC & GA & GM \\
\hline VBP, MDO & - & - & - & - \\
\hline Média da eficiência & 0,5174 & 0,5446 & 0,4812 & 0,5742 \\
\hline Acréscimo & $\mathbf{0 . 1 1 9 2}$ & 0.0262 & 0.0896 & 0.0624 \\
\hline
\end{tabular}

Fonte: Resultados da Pesquisa.

Quadro 4A. Escolha da Terceira variável, talhões não-irrigados - I-O Stepwise.

\begin{tabular}{|c|c|c|c|}
\hline Input & GM & GA & EC \\
\hline VBP, MDO, GC & - & - & - \\
\hline Média da eficiência & 0.6258 & 0.6430 & 0.6134 \\
\hline Acréscimo & 0.0516 & $\mathbf{0 . 0 6 8 7}$ & 0.0392 \\
\hline
\end{tabular}

Fonte: Resultados da Pesquisa. 
Quadro 5A. Escolha da quarta variável, talhões não-irrigados - I-O Stepwise.

\begin{tabular}{|c|c|c|}
\hline Input & EG & GM \\
\hline VBP, MDO, GC, GA & - & - \\
\hline Média da eficiência & 0.6716 & 0.6574 \\
\hline Acréscimo & $\mathbf{0 . 0 2 8 6}$ & 0.0014 \\
\hline
\end{tabular}

Fonte: Resultados da Pesquisa.

Quadro 6A. Escolha da primeira variável, talhões irrigados - I-O Stepwise.

\begin{tabular}{|c|c|c|c|c|c|}
\hline Input & GC & EC & GA & MDO & GM \\
\hline Output & VBP & VBP & VBP & VBP & VBP \\
\hline Média da eficiência & 0.3483 & 0.2970 & 0.4086 & $\mathbf{0 . 4 1 3 9}$ & 0.2905 \\
\hline
\end{tabular}

Fonte: Resultados da Pesquisa.

Nota: $\mathrm{GM}=$ Gasto com Manejo (controle de pragas e doenças; e controle de plantas daninhas), em R\$

Quadro 7A. Escolha da Segunda variável, talhões irrigados - I-O Stepwise.

\begin{tabular}{|c|c|c|c|c|}
\hline Input & GC & EC & GA & GM \\
\hline VBP, MDO & - & - & - & - \\
\hline Média da eficiência & 0.6200 & 0.4154 & 0.4791 & 0.4526 \\
\hline Acréscimo & $\mathbf{0 . 2 0 6 0}$ & 0.0014 & 0.0652 & 0.0387 \\
\hline
\end{tabular}

Fonte: Resultados da Pesquisa.

Quadro 8A. Escolha da Terceira variável, talhões irrigados - I-O Stepwise.

\begin{tabular}{|l|l|l|l|}
\hline Input & GM & GA & EC \\
\hline VBP, MDO, GC & - & - & - \\
\hline Média da eficiência & 0.6365 & 0.6412 & 0.6574 \\
\hline Acréscimo & 0.0166 & 0.0212 & $\mathbf{0 . 0 3 7 4}$ \\
\hline
\end{tabular}

Fonte: Resultados da Pesquisa.

Quadro 9A. Escolha da quarta variável, talhões irrigados - I-O Stepwise.

\begin{tabular}{|c|c|c|}
\hline Input & GA & GM \\
\hline VBP, MDO, GC, EG & - & - \\
\hline Média da eficiência & $\mathbf{0 . 6 7 1 1}$ & 0.6677 \\
\hline Acréscimo & 0.0184 & 0.0015 \\
\hline
\end{tabular}

Fonte: Resultados da Pesquisa. 
Tabela 1A. Teste de Wald de exogeneidade dos talhões não-irrigados, 2004/2006.

\begin{tabular}{lcc}
\hline Variáveis & Coeficiente & Erro-padrão \\
\hline Constante & 0,1523965 & 7,374615 \\
Prod. MDO & $-0,1765521$ & 0,1751011 \\
Prod. Terra & 0,0749922 & 0,2310144 \\
Capital & $6.36 \mathrm{e}-07$ & $5,87 \mathrm{e}-06$ \\
Prod. COE & 23,37185 & 150,2676 \\
\hline
\end{tabular}

Teste de Wald de exogeneidade: $\chi^{2}$ calculado $=0,20(p$-valor $=0,5320)$

Fonte: Resultados da pesquisa.

Tabela 2A. Teste de Hausman de endogeneidade dos talhões não-irrigados, 2004/2006.

\begin{tabular}{lcc}
\hline Variáveis & Coeficiente (Diferença) & Erro-padrão \\
\hline Prod. MDO & $-0,1115876$ & 0,1741856 \\
Prod. Terra & 0,0889259 & 0,2308797 \\
Capital & $8,57 \mathrm{e}-07$ & $5,73 \mathrm{e}-06$ \\
Prod. COE & 22,09391 & 0,1741856 \\
\hline
\end{tabular}

Teste de Hausman de endogeneidade: $\chi^{2}$ calculado $=0,88$ (p-valor $\left.=0,8305\right)$

Fonte: Resultados da pesquisa.

Tabela 3A. Teste de Wald de exogeneidade dos talhões irrigados, 2004/2006.

\begin{tabular}{lcc}
\hline Variáveis & Coeficiente & Erro-padrão \\
\hline Constante & 1,464712 & 1,037878 \\
Prod. MDO & 0,0063172 & 0,0461605 \\
Prod. Terra & $-0,0480753$ & 0,0274023 \\
Capital & $-1,09 \mathrm{e}-06$ & $1,02 \mathrm{e}-06$ \\
Prod. COE & 172,7614 & 210,6733 \\
\hline
\end{tabular}

Teste de Wald de exogeneidade: $\chi^{2}$ calculado $=20,29(p$-valor $=0,000)$

Fonte: Resultados da pesquisa.

Tabela 4A. Teste de Hausman de endogeneidade dos talhões irrigados, 2004/2006.

\begin{tabular}{lcc}
\hline Variáveis & Coeficiente (Diferença) & Erro-padrão \\
\hline Prod. MDO & 0,0617989 & 0,0418159 \\
Prod. Terra & $-0,0504894$ & 0,0268483 \\
Capital & $6,23 \mathrm{e}-07$ & $8,39 \mathrm{e}-07$ \\
Prod. COE & 361,0739 & 206,1433 \\
\hline
\end{tabular}

Teste de Hausman de endogeneidade: $\chi^{2}$ calculado $=5,23(p$-valor $=0,1554)$

Fonte: Resultados da pesquisa. 